\title{
Progress in research towards a world without leprosy. Report of a WHO meeting in Ethiopia, February 1998
}

\author{
P. ROCHE*, H. DOCKRELL** \& P. BRENNAN $\dagger$ \\ *Mycobacterial Research Laboratory, Anandaban Leprosy Hospital, \\ Kathmandu, Nepal \\ **Department of Infectious and Tropical Diseases, London School of \\ Hygiene \& Tropical Medicine, London, UK and †Department of \\ Microbiology, Colorado State University, USA
}

Accepted for publication 20 April 1998

Summary A UNDP/World Bank/WHO Special Programme for Research and Training in Tropical Diseases meeting to discuss the future role of biomedical research in leprosy, was held at the Armauer Hansen Research Institute in Addis Ababa, on February 27 and 28, 1998. This was attended by more than 20 scientists from 10 countries, who met to discuss progress towards a world without leprosy.

\section{Leprosy research in the context of immediate leprosy control needs}

The meeting was opened by the Chairman of the Board of Trustees of AHRI, who stated that despite the enormous progress made in leprosy control over the last 15 years, new cases of leprosy were still being detected, and that there was a real danger of leprosy research becoming marginalized in favour of TB research.

Dr D. B. Young (St. Mary's Hospital, London) reviewed the challenge that the success of chemotherapy poses for leprosy research. It is important that priorities are set for leprosy research which will support the leprosy elimination programme. These would include the development of tests for leprosy exposure (both skin tests and simple blood tests), tests for the prediction of reactions and better means of prevention of nerve damage. In the longer term, research could provide tools for surveillance of transmission, reactivation of disease, detection of non-human sources of infection and emergence of drug-resistant leprosy strains. Active preventative interventions such as chemoprophylaxis or vaccination of 'at risk' groups, identified by further research, would further reduce the incidence of leprosy. The lessons learnt from leprosy will not only benefit the patient, but provide further insights into the basic process of bacterial genetics, neurobiology and immunology.

Professor P. J. Brennan (Colorado State University) reminded the meeting of the priorities for leprosy research set at a meeting held in Bangkok in 1996, and also by the 7th WHO 
Expert Committee on Leprosy, and the ILEP Medical Commission. These meetings and groups addressed leprosy research from differing viewpoints, including those of individuals working on the genome and immunology of leprosy, the field workers who need tests for detection of early infection, and those who are involved in patient care and management. The purpose of the present meeting was to define further the agenda for biomedical research and to review progress toward the goals set in these previous meetings. The need to conserve resources such as people, laboratories, materials and techniques, training, and funding in leprosy research was also highlighted.

Dr S. K. Noordeen (WHO, Geneva) reviewed the role of research in the fight against leprosy over the past 25 years and suggested three priority areas from the perspective of the leprosy elimination campaign. These were a better understanding of and prevention of reactions and nerve damage, the detection and diagnosis of early leprosy, and the development of simple tools to detect drug-resistant leprosy. The emphasis may need to shift from the broad application of basic care to leprosy patients to a better management of the complications that leprosy causes in a large proportion of patients during and after chemotherapy. Dr Noordeen expressed his optimism that, despite the shrinking size of the leprosy research community, these new tools will be forthcoming in the short term.

Dr W. C. Smith (ILEP, London) gave a view of research needs from an epidemiological perspective. He stressed the need to maintain the dialogue between research scientists, control programmes and epidemiologists and reminded the audience of the difficulties of applying new tests into leprosy care when vertical leprosy programmes have largely been integrated into primary health care services. A number of the current priorities for epidemiological research have biomedical implications. These include the need to measure and monitor leprosy incidence by a more accurate surrogate measure than the new case detection rate, which can reflect the intensity of the leprosy control effort rather than actual disease transmission. The development of simulation models to predict future trends in leprosy incidence under varying scenarios, the evaluation of the implication of detection of Mycobacterium leprae in nasal swabs from unaffected individuals and the definition of high risk communities who would benefit from active prophylactic regimens are all areas which require new biomedical tools.

\section{Serology}

Dr P. Klatser (Royal Tropical Institute, Amsterdam) reviewed recent serological data from work in Indonesia, which indicate that household contacts of leprosy patients who are seropositive for IgM anti-phenolic glycolipid I (PGL-1) antibodies have an eight-fold higher risk of developing leprosy. Dr Klatser also presented data from a 25-year survey of leprosy cases in an Indonesian village which showed that almost all of the incident cases had some kind of contact (household, work or social) with active leprosy cases. This group of 'contacts' will tend to become the dominant group from which new cases arise as the incidence of leprosy falls and serology can be a valuable tool to identify those at highest risk. Moreover, measurements of anti-PGL-1 antibodies can now be done using a simple 'dipstick' which is cheap (costing about US $\$ 1 /$ test), reliable, and well suited to field use. Operational studies in various centres to test the predictive value of the dipstick test should be started shortly. The identification of these seropositive contacts has the potential to identify most of the incident cases of leprosy at a subclinical stage of the disease. 
Dr R. Hussain (Aga Khan University, Karachi) emphasized the important information that serological measurements in leprosy patients can give to predict the severity of disease, the response to chemotherapy, the onset of reactions and relapses. The profile of antibody classes and subclasses found in leprosy is not characteristic of the Th2 activation seen in allergic disease or helminth infection, as it is IgG1 and IgG3 antibodies, rather than IgG4, that correlate with the bacterial index. Areas needing further research include the role of cytokines in antibody class and subclass switching in man, and the interaction of these IgG subclass antibodies and immune complexes with macrophage Fc receptors. Many leprosy contacts make an early antibody response to $M$. leprae, and the precise pattern of antibodies and cytokines induced may be predictive of the eventual development of infection, or protection.

\section{Gene amplification techniques}

Dr S. Cole (Institute Pasteur, Paris) reviewed the applications of gene amplification techniques to leprosy. Despite grandiose claims for applications of polymerase chain reaction (PCR) technology to leprosy, only a few well conducted studies have assessed the reliability of PCR in detecting leprosy in clinical samples. A multi-centre study in 1994 showed good sensitivity, specificity and reproducibility, using a standardized protocol based on the RLEP sequence. Dr Cole suggested three applications of this technology - to the diagnosis of rare or difficult cases, the diagnosis of drug resistance (to current and future anti-leprosy drugs, such as rifampicin, ofloxacin and minocycline), and the definition of relapse as reactivation or new infection by means of molecular strain typing of $M$. leprae in clinical samples. A further application presently under investigation is the nasal carriage of $M$. leprae by unaffected persons. Studies in India and Ethiopia find a higher rate of nasal $M$. leprae carriage (4.7\%) than predicted from the prevalence rates (W. C. Smith) and such carriers may have a higher risk of developing clinical leprosy.

\section{Skin tests and simplified whole blood tests to measure leprosy exposure}

Dr H. M. Dockrell (London School of Hygiene \& Tropical Medicine) described the first of two new initiatives which hope to develop new leprosy-specific skin test reagents. Four centres in leprosy endemic countries have been testing 193 peptides for their ability to induce $\mathrm{T}$ cell proliferation and IFN $\gamma$ secretion in peripheral blood mononuclear cells (PBMC) from leprosy patients and healthy leprosy contacts, in a study initiated by the WHO IMMYC Steering Committee. These peptide sequences (15 amino acids in length), which were derived from the genome sequencing data now available for $M$. leprae, have been selected from known and unknown proteins which appear leprosy-specific and contain putative HLA-DR binding motifs. Initially, the peptides were tested in 19 pools, each containing 10-11 peptides, most of which elicited strong $\mathrm{T}$ cell responses. The individual peptides from eight pools will now be tested, with the hope that a highly leprosy-specific peptide based diagnostic test will result.

Dr P. J. Brennan (Colorado State University) discussed another approach to the development of a leprosy-specific skin test, involving the fractionation of the leprosy bacteria into cytosolic and cell-wall protein fractions, free of immunosuppressive lipid and carbohydrate moieties. The production of these fractions under good manufacturing practice 
conditions has been achieved and phase I safety trials in the USA will occur in the autumn of 1998; following certification by the Food and Drug Administration the fractions should be used as skin tests in the field in early 1999. Even if these reagents are not suitable for individual diagnosis, they may be very valuable for epidemiological studies on trends in leprosy transmission, and with further fractionation, may also yield a highly specific diagnostic skin test.

Simplified blood cultures using unfractionated whole blood stimulated by the new leprosy antigenic fractions have been shown to give proliferation and interferon-gamma production equivalent to that shown by peripheral blood mononuclear cells (Dr H. M. Dockrell, London School of Hygiene \& Tropical Medicine). The new fractions appear more potent than earlier $M$. leprae sonicates, but further fractionation may be required to achieve the required specificity, as both human and guinea pig experiments indicate that at present the fractions are cross-reactive with antigens in $M$. bovis BCG and $M$. tuberculosis. Studies in TB patients have shown a good correlation between PDD-induced interferon-gamma production in whole blood cultures and skin test reactivity to tuberculin (Dr R. Hussain, Aga Khan University). These simple blood tests may as easy to use in the field as skin tests, as although a basic laboratory is needed, the subject does not need to be recalled for the skin test to be read.

Dr Sengupta (JALMA, Agra, India) and Dr Jim Krahenbuhl (National Hansen's Disease Center, USA) led to a discussion on the role of laboratories in the field diagnosis of leprosy. It is not clear how necessary central laboratories, run by national control programmes, are for the effective running of control programmes, but there is clearly a need for continued training and quality control as leprosy moves towards the post-elimination era. It is important that the skills involved in leprosy diagnosis should not be lost, as even in non-endemic countries occasional leprosy patients need to be accurately diagnosed. As leprosy cases decline, it may also be appropriate to establish central banks of leprosy sera, biopsies, or cells, as a resource for the research community.

\section{Leprosy reactions and nerve damage}

Dr G. Kaplan (Rockefeller University, USA) introduced the second day's programme, on future directions in leprosy research, by highlighting some of the unique features of leprosy. These include the development of reactions and nerve damage, which can occur after the completion of treatment and microbiological 'cure'. Nerve damage and reactional complications will remain a problem for leprosy patients even in the post-elimination era. The new knowledge available from the $M$. leprae genome should now allow the identification of the unique features of $M$. leprae. It should also be possible to exploit recent developments in neurobiology and immunology to identify those individuals who will develop nerve damage. Genetic analyses will also facilitate the prediction of which patients are at risk of developing reactions.

Dr E. Sampaio (Fundacao Oswaldo Cruz, Rio de Janeiro) reviewed recent work on the pathology of erythema nodosum leprosum (ENL), mediated by the cytokine tumour necrosis factor alpha $(\mathrm{TNF} \alpha)$. High levels of serum TNF have been found to be predictive of ENL reactions and are possibly associated with the development of disability. TNF $\alpha$ is produced by monocytes and macrophages, and both IFN $\gamma$ and membrane contact between lymphocytes and monocytes increases TNF production. There are also genetic polymorphisms in the TNF $\alpha$ promoter region which may affect an individual's capacity to make TNF. Further work 
on the regulation of TNF, its role in nerve damage and the identification of patients at high risk of reactions is underway.

Dr G. Kaplan (The Rockefeller University, New York), discussed the mechanisms of ENL, and its treatment with thalidomide. Thalidomide has been known for many years to be effective as a treatment of ENL, but the mechanism of its action was not clear. As high levels of $\mathrm{TNF} \alpha$ are present in the plasma of patients with ENL, it was possible that thalidomide might affect $\mathrm{TNF} \alpha$ production. Thalidomide has been shown to inhibit the production of $\mathrm{TNF} \alpha$, which is found at high levels in the plasma of ENL patients, but not that of other cytokines such as IL-1, IL-6 or GM-CSF. Since TNF $\alpha$ can reproduce many of the systemic symptoms of ENL if injected into humans or animals, this cytokine has been proposed to play a central role in the pathogenesis of ENL. TNF $\alpha$ also plays a role in protective immunity: for example, it is required for granuloma formation, and it is therefore both helpful and harmful to the patients. Recently, thalidomide has been shown to induce stimulation of T cells, and in particular, the CD8+ T cell subset. It is hypothesized that thalidomide modulates the host response to mycobacterial infection not only by inhibiting $\mathrm{TNF} \alpha$ production but also by stimulating $\mathrm{T}$ cells to produce more of the Th1 type cytokines IL-2, IFN $\gamma$ and IL-12, thus resetting the extreme Th2 cytokine profile of ENL towards a Th1 cytokine profile. Recent work has led to the identification of thalidomide analogues which have enhanced TNF inhibitory activity, but which appear to lack the teratogenic action of thalidomide. It is hoped that some of these new thalidomide analogues might provide non-teratogenic treatments for both ENL and reversal reactions within the next 10 years.

Dr D. Lockwood (London School of Hygiene \& Tropical Medicine, UK) discussed new insights into the mechanisms of nerve damage in leprosy. There are at least three types of nerve damage, the acute reactions that occur in reversal (type 1) reactions and ENL, chronic neuritis, and quiet nerve paralysis which may account for the majority of nerve damage. Leprosy antigens can be detected within the nerve granulomas and Schwann cells, and may persist even following the completion of treatment. Both CD4 and CD8 T cells are present within affected nerves, with BT patients in reversal reaction showing the highest CD4/CD8 ratio. Both $\mathrm{TNF} \alpha$ mRNA and protein can be detected within nerve granulomas during reversal reactions. There are also increases in the expression of adhesion molecules, inducible nitric oxide synthase (iNOS), IL-2R and IFN $\gamma$, suggesting stimulation of Th1 T cells. The need for a more rational treatment of type 1 reactions was stressed as more than one-third of patients do not respond to corticosteroids. New drugs for trial in the treatment of reversal reactions include azathioprine and cyclosporin $\mathrm{A}$, which are available now and the thalidomide analogues, TNF $\alpha$ antibodies and inhibitors of iNOS which should be available for testing in the future. Future research into the molecular pathogenesis of reversal reactions and the reliable measurement of nerve damage should lead to more effective treatments for type 1 reactions, which will result in reduced disability in leprosy.

Dr Rambukhana (The Rockefeller University, New York, USA) discussed the mechanism of entry of M. leprae into Schwann cells. M. leprae was shown to bind to laminin, present in the basal lamina of Schwann cells, but not to fibronectin. Laminin exists in a number of isoforms, and it is the laminin-2 form ( $\alpha 2 \beta 1 \gamma 1)$ which is expressed by cultured Schwann cells. Within the laminin- $2 \alpha 2$ chain, the G2 domain is critical for $M$. leprae binding. If the $\alpha 2$ chain of laminin-2 is missing, as in the $d y / d y$ mouse model of muscular dystrophy, M. leprae binding, and the subsequent rearrangement of the cell cytoskeleton to allow bacterial entry, do not occur. This is the first major advance using tools of molecular and cellular biology to understand this unique feature of the leprosy bacteria. Further work may provide new 
therapeutic strategies by blocking $M$. leprae uptake with competitive peptides, or other blocking agents.

\section{Drug resistance}

Dr P. Roche (Anandaban Leprosy Hospital, Kathmandu) discussed drug resistance in leprosy. The current prevalence of resistance to the three drugs used in the MDT regime is unknown but is thought to be low. Relapses after MDT treatment are rare and seem to be the result of reactivation of drug-sensitive organisms; thus retreatment with MDT is usually successful. The mouse foot pad assay, despite being costly and time-consuming, remains the 'gold standard' for demonstrating drug resistance. New insights from genetic studies have allowed the development of a new gene-based method to detect rifampicin-resistant leprosy. Because of the importance of rifampicin to the success of MDT and rifampicin/ofloxacin/minocycline (ROM) regimens, the monitoring of rifampicin resistance by gene-based assays is considered vital. A pilot study, funded by IMMYC has commenced in India and Nepal to measure the prevalence of rifampicin resistance among MDT relapses using a PCR based line-probe assay developed by Dr S. Cole (Institut Pasteur, Paris). This study will investigate the relationship between the detection of rifampicin resistant mutants in such patients and the response to (re)treatment. The sequencing of the gene associated with rifampicin resistance (rpoB) from all these $M$. leprae isolates may also identify other mutations which may give rise to rifampicin resistance.

\section{Genomics}

Dr Stewart Cole (Institut Pasteur, Paris) described the latest developments in the M. leprae genome sequencing project. Genomics, the study of the genome, aims to identify all the genes and regulatory sequences that are present, and deduce the functions of these genes using bioinformatics. Since an ordered library of M. leprae cosmids became available in 1991, sequencing of the $M$. leprae genome has been underway at the Pasteur Institute, Genome Therapeutics Corporation and the Sanger Centre, and $95 \%$ of the genome has now been sequenced.

The preliminary analysis of the genome reveals some surprises, particularly as compared with the recently completed $M$. tuberculosis genome. The $M$. leprae genome is $50 \%$ smaller than that of $M$. tuberculosis, and many of the genes present in $M$. tuberculosis have been lost from $M$. leprae. A broad classification of the genes of $M$. leprae into four groups reveals about 1100 house-keeping genes, 300 genes which encode proteins common to other mycobacteria, 50 genes for exported proteins, which would include those inducing protective immunity, and about 60 unique proteins which will be useful for understanding the biology of M. leprae and for use as leprosy diagnostics. Data from the genome sequencing initiative are available to investigators via the MycDB database, both in a 'stand alone' UNIX format and on the World Wide Web. The MycDB database has links to other databases such as SwissProt, EMBL and Medline. This valuable database needs to be maintained and improved to gain the maximum benefit from the $M$. leprae sequencing data.

Dr Antoine Danchin (Institut Pasteur, France) who has worked on the B. subtilis genome, talked about the potential of such genome information. Genome mapping is an increasingly 
common activity in microbiology, with the publication almost monthly of complete genomes from bacterial species; about 300 are expected to be completed over the next decade. Virulent bacteria have often captured extra genetic material, the genes for which may be located on pathogenicity islands. Bacteria can also occupy different biotopes, and have distinct survival strategies, which can lead to individual features or 'styles'. Much information will be obtained by 'in silico' experiments, in which sequence data are analysed for patterns of codon usage, GC content and the distribution of coding regions, TA/GA dinucleotides and the predicted isoelectric point (pI) of encoded proteins. The structure of the genome reveals much about the adaptive biology of the organism and comparisons with other mycobacteria can lead to much useful information on drug targets and immunology.

\section{New vaccines for leprosy?}

A round table discussion on the future of leprosy vaccines was led by Dr J. Krahenbuhl, (National Hansen's Disease Center, USA). The large Karonga Prevention Trial in Malawi demonstrated that $\mathrm{BCG}$ vaccination induced $50 \%$ protection against leprosy, and that the addition of killed $M$. leprae to BCG did not enhance this protection. It seems unlikely that much effort will now be put into the development of new anti-leprosy vaccines, but new vaccines against tuberculosis are in development, and these might confer even greater protection against leprosy than BCG. It will therefore be important to test such candidate tuberculosis vaccines for their ability to protect against $M$. leprae in the mouse foot-pad model. From the field perspective, Dr Noordeen confirmed that it would now be too costly to implement a total coverage vaccine against leprosy, but vaccines with protective efficacy against leprosy could be important as adjuncts to chemotherapy, or for use in high risk groups or areas.

\section{Short term and long term priorities for leprosy research}

Dr P. Brennan (Colorado State University, USA) then summarized the priorities identified for leprosy research in both the short term (the next 5 years) and long term (5-10 years). In 10 years time, leprosy will occur at low prevalence especially in isolated communities beyond the reach of control programmes. There is therefore still a need to train new people who will contribute to the final eradication of leprosy as a public health problem. Our lack of understanding about the transmission of $M$. leprae, and whether it has an environmental reservoir, means that even as the number of cases of leprosy fall, there is a real need for continued surveillance. New tools which will have direct practical benefit for the control programme include tests to identify 'at risk' contacts by serology, the use of PCR to detect rifampicin resistance, the development of skin tests and whole blood tests to measure leprosy exposure and new treatments for leprosy reactions. It is also important that facilities such as the mouse foot pad laboratories, slit skin smear services and banks of clinical specimens should be preserved.

Overall, leprosy research is currently in a healthy state, with strong research groups and leadership in both research and control areas. New leprosy research is not required for the current leprosy elimination programme, but it can and should play an important role in the post-elimination era by providing new field-applicable tools to identify areas or groups at 
high risk, and in monitoring progress towards the eradication of this debilitating disease. These may include new dipstick assays for antibodies or leprosy antigens, whole blood assays for cellular responses, specific skin test reagents, and PCR tests for the detection of nasal carriers of $M$. leprae. The new knowledge available from the $M$. leprae genome will also lead to new insights into why $M$. leprae behaves as it does, with its predilection for nerves, and its capacity to induce anergy in a proportion of those it infects. Understanding why some individuals develop reactions, and how these can be controlled will also provide new information on the molecular interactions occurring in immunopathology. These challenges will keep scientists absorbed by leprosy and producing insights which will have a fundamental impact in bacteriology, neurology and immunology.

\section{Conclusions and recommendations}

1. The decline in leprosy patients and the commitment to leprosy elimination as a public health problem have been matched by a decline in leprosy research, with the majority of active researchers turning to tuberculosis research. Nevertheless, there is a strong core of active investigators, central laboratories, and shared resources. The retention of this pool of expertise and resources is crucial.

2. The discussion of research priorities should be a continuing proposition, and flexible short-term (5 years) and long-term (10 years) global research plans should be defined.

3. In immediate terms, it is unlikely that biomedical research will help in leprosy control. Rather, the agenda should include alternative measures for leprosy control in support of current efforts and bearing in mind the possibility of a breakdown in the elimination program in future years. Control programs should recognize the considerable achievements in research, particularly in diagnosis, and incorporate them into leprosy control measures, especially to the detection of leprosy in isolated populations in the postelimination period.

4. The full sequence of the $M$. leprae genome and the closure of gaps is a priority in the short term. A concerted effort to express, purify and implement for diagnostic purposes, the full range of $M$. leprae-specific protein and the family of export proteins is a priority. This effort will require co-operation among several global laboratories and sizeable funding.

5. Considerable progress has been made in the simplification of serodiagnosis and application to important clinical issues. Efforts should continue to enhance sensitivity to allow better diagnosis at the tuberculoid end of the disease. Likewise, with PCR and whole blood assays for cytokines. Skin test antigens offer the best prospect for large-scale monitoring of leprosy in a community, and their continuing development is a priority.

6. The preservation of central global leprosy research laboratories is a priority to allow the preservation of expertise and resources for technology such as acid fast staining, skin slit smear, ELISA, dot-blot, PCR, mouse footpad, cytokine profiling, and banks of bacilli antigens, antibodies, cosmids, databases, etc.

7. The continuation of research on the treatment of neuritis and reactions and on the early diagnosis of reactions, is a priority.

In the long term:

1. The preservation of expertise, resources and fundamental research on the immunology of 
leprosy, the molecular and immunological basis of the tropism of M. leprae for Schwann cells and subsequent damage, the physiology and genetics of the organism, are all crucial. The polarity of the immune response seen in leprosy requires understanding.

2. Experience with tuberculosis has taught us the consequences of the dismantling of specialized research on a major disease. Hence, the preservation of personnel, laboratories and resources is important.

3. The complete eradication of leprosy is unlikely through present measures. The development of a vaccine will always be a priority. However, the plan should be more passive, waiting to see what basic research on the immunology and bacteriology of leprosy will bring, in addition to exploiting the extensive research on tuberculosis vaccine development. 